\title{
ESTUDIO PRELIMINAR SOBRE LOS FACTORES INFLUYENTES EN LA ABSORCION AEREA Y TRANSPORTE DE ELEMENTOS NUTRITIVOS (1)
}

\author{
POR RUBFN O. MENINATO
}

\section{CONSIDERACIONES GenERALEs sobre absorclón Fuliar}

Es, probablemente, tan viejo como la misma agricultura, el conorimiento de que las raíces son los órganos de la planta ruya función es la absorción del agua y los nutrientes. Sin embargo, dosde hace tiempo, existen obserraciones indicatiras de que las plantas pueden absorber nutrientes por las partes aéreas, vale decir hojas, ramas, brotes y troncos, siendo interesante destacar que las primeras referencias relativas a este proceso datan del siglo xvm, (Miller, Garlener,s, Dictionary, 17j4, Downin, 1869, Foreyth, 1803).

En los últimos años los radioisotopos permitieron un estudio exhaustivo de la absorción y transporte, al hacer posible la retección o marcación de los nutrientes diferenciándolos de los que la planta hubier: absorbido por diferentes procesos.

La necesidad de apuítar clatos más precisos acerca do los procesos cle absorción y transporte se vió aumentada por nuevos proceclimientos que trajeron como consecuencia cambios profundos sobre murhas prácticas agrícolas. Del interés por los procesos mencionados coparticipan estudiosos en materias como plaguicidas, especialmente los de tipo sistémiro, hormonas, como el tan divulgado 2, 4D y otras. antibióticos, fertiliclad y en general aquellos ligados a la apliración de estos productos por ser también de importancia la forma y tipo de aspersión.

En materia de fertilización nuevos conceptos, forzaclos en parte por mercados crecientes en demanda de caliclad y cantidad, uniclo a lo ajustado de la relación costo-precio, que caracteriza al momento agrícola actual, obligaron a procedimientos más exartos y, muchas veces, a revaluar conorimientos de tipo rlásico.

\footnotetext{
${ }^{1}$ Lis observacion's ef etuadas con $z_{n}^{65}$ se llevaron a cabo on el Departamento de Acidioisoupos del Instituto Intrimericano de Ciencias Agrícolas de Turrialba, Costa lica, sobre plantas ivenes de café Tienen caráctar de entrenmiento y se deseriben a título ilustrativo.
} 
La absorción de nutrientes por vía foliar es tema de especial interés para los interesaclos en problemas de fertilidad cuyo objetivo inmediato es el aumento de las cosechas por el uso de fertilizantes cunstituyendo, en este campo. las aspersiones nutritivas nuevos procedimientos que deberán cumplementar los tratamientos de suelo.

\section{Factores de influencia en i.a Absorción Foliar}

Numerosos factores pueden influenciar la absorción de los nutrientes aplicados por vía foliar. Según Karnd ( $\left.{ }^{1}\right)$ los mismos pueden clasificarse en la siguiente forma:

a) Físicos y químicos de nutrición.

1) Naturaleza de la sal usada como nutriente.

2) Concentrarión de nutriente.

b) Biológicos Puramente.

3) La planta.

4) Estado de desarrollo de la planta.

5) Ritmo de crecimiento anual.

c) Interacción entre nutrientes y planta.

6) Cantidad de nutriente aplicarlo.

7) Tiempo de acción del nutriente.

8) Lugar de aplicación en la hoja.

d) Condiciones del medio.

9) Humedad y formación de rocío.

10) Temperatura.

11) Hora del clía.

12) Calidad del suelo.

Si bien el estudio indicado se refiere a absorión foliar es oportuno destacar que en la misma está comprendida la realizada pur otras partes de la planta (brotes, ramas, tronco) por lo que corresponde referir a absurción aérea como término que involucre el proceso mencionado $\left({ }^{2}\right),\left({ }^{3}\right),\left({ }^{4}\right)$.

Numerosos trabajos han estucliado los factores de influencia en la absorción y, en algunos casos, lo que podría denominarse segunda parte del proceso; el transporte de translocación del nutriente.

Wallihan y HeymanN-HerschberG $\left({ }^{5}\right)$, por ejemplo, refirren que el Zn fué absorbido y trasladado más rápidamente: 1) cuando fué aplicarlo a hojas jóvenes, 2) a altas concentraciones de Zn por unidad de área, 3) cerca del centro de la hoja.

Wallace y BEDRI $\left(^{(5)}\right.$ destaran que las aspersiones de urea, en huertos citrícolas, trajeron un mejor aprovechamiento de quelato 
Fe (EDDHA), mientras que redujo la absorción de los compuestos de Zn usados. A su vez el agregado de aceite aumentó la absorción cle Fe y disminuyó la de $\mathrm{Zn}$. Destacan asimismo que en general la traslación de los elementos Zn y Fe, a otras partes de la planta, fué función de la cantidad absorbida.

TeubNer y otros observan que las hojas de arvejas son más retentivas y absorben más rápido el fósforo que las de tomate. Mayor absorción durante el día o con luz. Disminución de absorción por presencia de sacarosa en la solución. Temperatura óptima de 21" para la absorción clel fósforo y 25 a $30^{\circ}$ para la del potasio. Máxima absorción del fosfato a $\mathrm{pH} 2$ a 3 con amonio como ión portador. La acción de algunos humectantes rerluciendo la adherencia y disminuyenclo la absoinción. Que altos niveles de fosfato en la raíz deprimen el total porcentaje derivado del tratamiento foliar $\left({ }^{7}\right)$.

Gustafson a su vez encuentra: en general incremento de absorción con luz y temperatura, haciendo notar una exrepción en el comportamiento del 2, 4D en jacintos, al artuar en condiciones de falta de luz. Destaca también que plantas que han estaclo 24 a 72 horas a la sombra, absorben pero no trasladan. Observa aumentos de absorción por influencia del azúcar y los aceites $\left(^{8}\right)$.

Volk y McArliffe $\left({ }^{9}\right)$ comprueban que la absorción de urea, mircarla con $\mathrm{N}^{15}$, es en tabaco de tres a diez veces mayor por la noche. A su vez refieren a la sacarosa como factor limitante de la absorción la que procedería según las siguientes hipótesis: a) por impedir la difusión de la molécula (le urea, b) por competir con la urea por entrar en la planta, c) por inhibición de la activirlad de la ureasa.

Kalivd ( $\left.{ }^{1}\right)$ sostiene la dependencia de la absorción foliar con las etapas del desarrollo. Comprueba que una disminución de $7^{\circ} \mathrm{C}$ en la temperatura (23 a 16") a umentó al doble la absorción $\mathrm{y}$, por otra parte, que por la tarde fué más alto que por la mañana.

\section{Mecanismo de Absorctón}

Uno de los más discutidos factores relativo a la absorción es el referente a la estructura de la hoja que facilite o pueda correlacionarse con aquella. La vía de entrada del nutriente generalmente, por haberse efectuado en mayor proporción por la parte inferior de la hoja, a su vez coincidente en muchas especies con una mayor proporción de estomas, ha llevado, incluso a autores recientes a correlacionar absorción con número de estomas, y, en consecuencia a buscar en este mecanismo la explicación del proceso absorbente.

Este aspecto de la absorción, aún hoy no esclarecido completamente, permite en base a los conocimientos actuales negar la influencia estomática en el mismo.

Tuckey et al. (2) hacen notar que cuando se trata la hoja de 
habichuela ron una solución de $\mathrm{H}_{3} \mathrm{PO}_{4}$ radiactivo, la absorción por' la superficie es algo mayor. Dado a que la densidad de estomas es siete veces mayur en la cara inferior, desprende que la absorción del fosfato no es facilitada por tales estructuras.

Gustafson $\left(^{8}\right)$, en un trabajo entregado a publicar en 1955 destacaba la influencia estomática relacionando una mayor absorción con la hoja turgente y correlación entre posición de estomas y absorción. El mismo autor $\left(^{10}\right)$ destaraba un año después que algunas plantas absorben preferentemente por la superficie superior y otras por la inferior. Esta diferencia, ahora necesita ser asociarla con los estomas. La cutícula es considerada más delgada en la parte inferior y esta diferencia porría ser el factor que acrece la absorción.

Sin embargo, estudios realizados en 1933 ('1) al conientar la influencia del $\mathrm{H}_{2} \mathrm{SO}_{4}$ como agente penetrante en aspersiones arsenicales para control de maleza indicaban que la cutícula en muchas hojas es fácilmente permeable al vapor de agua y moléculas disueltas, siendo la entrada por estomas, ínfima o sin dur lugar a penetración.

La discontinuidad de la cutina (12) como factor de absorción es destacada en diversos estudios $\left({ }^{13}\right)$ y los principales factores que influirían sobre la misma serían: pubescencia, humectantes y las variaciones propias de la cutícula.

Investigaciones recientes acuerdan importancia en el proceso absorbente a los plasmodesmos conjuntamente con la irregularidad de cutícula $\left({ }^{14}\right)$.

Los plasmodesmas son filamentos plasmáticos que a través de. los canalículos de las membranas separatrices celulares ponen en romunicación dus células contiguas haciendo perdurable la primitira continuidad $\left({ }^{15}\right)\left({ }^{16}\right)$.

Asimismo leves modificaciones de la estructura foliar pueden tener consecuencias significativas en la absorción, por ejemplo, el cepillado de hojas de tabaco ${ }^{\left({ }^{9}\right)}$ promovió la absorción al quebrar prolongaciones epicérmicas cubiertas por cutina, y, poner en contacto directamente, a la solución con la epiclermis.

En otro caso $\left(^{1}\right)$, en hojas de Sulanum nigrum, se comprobó que la cara inferior absorbe el doble que la superior pero poseyento un $50 \%$ más de superficie.

Pueden interesar en un estudio sobre estruaturas de absormión en plantas de café, por ejemplo, cletalles anatómicos que quizá resulten significativos en el proceso. Esta especie absorbe más por la cara inferior cle la hoja (17,) y presenta en la misma poros redondos o elípticos que miden de 120 a 380 micrones, generalmente habitados por parásitos, siendo el nombre de nstos poros, domacios ( ${ }^{18}$ ).

Esta característica podría favorecer las discontinuidades y afinamientos de cutícula aumentados por las lesiones prorlucidas por los mismos parásitos furoreciéndose así el proceso de absorción. 


\section{iII. Factores influyentes en la Aspersión Nutritiva}

Puede verse así que el proceso de absorción es complejo y no exento de aparentes contradicciones. La planta, como material, desarrolla un papel fundamental dado a que muchas de las variantes le son especificas.

Los elementos, como veremos más adelante, tienen también características definidas que se ponen cle manifiesto fundamentalmente en el proceso de transporte o traslación.

Desde el punto de vista de fertilidad pueden deducirse algunas conclusiones:

a) Todas las substancias que la planta absorbe por la raíz puerlen ser también absorbirlas por hoja y con mayor eficiencia en la generalidad de los casos ( $\left.{ }^{1}\right)$.

b) La cantidad de elemento trasladado a otras partes de la planta es, en general, función del elemento absorbido $\left({ }^{6}\right)$.

Intentando enumerar los fartores a tener en cuenta en una aspersión nutritiva como consecuencia de lo expuesto tendríamos:

1) Una gran propor •ión de plantas absorben más sustancia por la parte inferior de sus hojas, aspecto que debe ser tenido en cuenta, en los sistemas de los aspersores a utilizar.

2) En general los humectantes, por disminuir la tensión superficial son de conveniente empleo.

3) Las aspersiones conviene realizarlas en horas de la tarde pues se favorece el secado lento y, por la mañana, el rocío removiliza las sustancias. Después de la aplicación de una aspersión nutritiva puede convenir asperjar con pequeñas cantidades agua. ${ }^{(1)}$.

4) Es importante tener presente la naturaleza de la sal a utilizar dado la significativa influencia del ión portador $\left(^{7}\right)$.

Lo expuesto hace llegar a la conclusión de que la absorción foliar es un proceso activo debido a numerosos factores, muchos de los cuales controlables, son fácilmente influenciables para los fines de la práctica agrícola y pueden conducir a una mayor efectividad de las aplicaciones.

La tendencia moderna es la de reducir los tratamientos $\mathrm{y}$, en consecuencia, dar a los mismos valores múltiples. Por ejemplo, la aplicación conjunta de varios nutrientes por vía foliar, y en muchos casos, unir a los mismos uno o más plaguicidas o productos hormonales.

Es de importancia, en consecuencia, ampliar la investigación relativa a absorción conduciéndola de acuerdo a los problemas que surgen de los distintos cultivos en base a sus requerimientos. Como 
hemos visto un determinado adhesivo puede ser de importancia al pormitir una mayor o menor absorción (4), la sacarosia y el boro, en algunos casos, han reducido la absorción de fósforo en hojas de habas $\left({ }^{2}\right)$. En trigo, contrariamente, se aumentó la absorción por medio de diversos azúcares. La urea contribuyó a aumentur la absorción de quelatos de Fe, y redujo la de los compuestos de Zn (6).

El uso de soluciones de zine y cobre, en citrus y tung respectivamente, aplicadas con cal (13) permiten la precipitación de los compuestos en las superficies foliares y una reducción de la absorción. Por otra parte, las necesidades de estos elementos para la planta son de un orden sumannente reducido y una gran absorción podría ocasionar perjuicios. La acción de la cal serviría para eliminar los mismos y asegurar una absorción reducida.

La acción de la urea asperjada en manzanos se incrementa con el uso de $\mathrm{SO}_{4} \mathrm{Mg}$. En tomate se observa que disminuyen los perjuicios que puede ocasionar una excesiva absurción de area adicionando $\mathrm{SO}_{4} \mathrm{Mg}$. Igualmente en otras especies los tratamientos con urea pueden ocasionar daños qie son controlados aplicanclo simultáneamente en algunos casos, como los cítricos, caldo bordelés y en otros, azúcares, que como hemos visto, pueden reducir la absorción de urea.

Numerosos ejemplos y extensa bibliografía puerte ampliar los datos expuestos destinados a resaltar la importancia que diversos factores pueden tener sobre el proceso de absorción.

\section{Traslación}

Una rápida revisión al tema movimiento o transporte de nutrientes absorbidos nos permite diferenciar dus grupos:

1) Los que se mueven libremente, aparentemente poro afertados por las condiciones nutritivas impuestas a la planta: ej. $\mathrm{N}$, F, S, K y Mg. Todos ellos pueden ser redistribuidos dentro de la planta cuando sobrevienen períodos de escasez.

2) Los que se mueven en muy pequeña cantidad y son fárilmente afectados por las condiciones nutritivas: ej. Ca, Fe y en cierto modo $\mathrm{Zn}\left({ }^{19}\right)$.

Este aspecto de la redistribución cle los elementos de la planta puede onstituir la clave de muchos tratamientos mediante aspersiones y explica la aparente ineficacia que en determinadas condiciones han resultado aplicaciones de $\mathrm{Ca}, \mathrm{Fe}$ y $\mathrm{Zn}$.

La comprensión del problema da las bases del diagnóstico foliar de problemas carenciales. Deficiencias de $\mathrm{N}, \mathrm{P}, \mathrm{K}, \mathrm{Mg}$ o $\mathrm{S}$ se encontrarán primero en las partes viejas de la planta mientras que las de $\mathrm{Ca}, \mathrm{Fe}$ o $\mathrm{Zn}$ comenzarán a manifestarse por los brotes u hojas tiernas $\left({ }^{20}\right)$. 
Solo la investigación y experimentación podrán esclarecer hasta que punto, por acción e interacción de factores, puede ser influenciada la tralación de los nutrientes clentro de la planta.

\section{Fertilización AÉrea y por incorporación al suelo}

La nutrición foliar se ha impuesto como solución de problemas carenciales en aquellos casos que la ahsorción por raíz se vió impedida por razones de escasez, insolubilidad o, en general, falta de disponibilidad rlel elemento.

Los progrusos alcanzarlos en el campo cle la fertilización clásica, incorporando los nutrientes al suelo, permiten discutir la hipótesis de si asegurando nutrientes rápidamente asequibles podrá llegarse a bastar las necesirlades de las plantas prescindiendo así de los tratamientos aéreos. Sería el caso cle los quelatos que en determinadas rondiciones han resuelto problemas de deficiencias cuya única solución, antes de ser utilizarlos, era la aspersión foliar le sales ionizables. El ejemplo sería aplicable a algunos micronutrientes.

En cuanto a los elementos de gran consumo por parte de la planta corresponde definir hasta que punto la investigación acerca de sus pusibilidades de ser incorporarlos por hoja no deja de tener un interés puramente teórico.

Respecto al primer caso, el de micronutrientes, el uso de quelatos se ha visto limitarlo en muchos casos por consideraciones económicas $\left(^{6}\right)$ y por problemas provenientes de la composición y reacción del suelo que en determinadas condiciones no permiten el aprovechamiento de ciertos quelatos (21). La aspersión foliar de uno o muy pocos tratamientos puede bastar para llenar las necesidades de la planta y si estos pueden ser combinados con aplicaciones de tipo sanitario u hormonal llegamos a la conclusión de que dificilmente se podrán encontrar procedimientos más ventajosos.

En aquellos nutrientes que como el $\mathrm{N}, \mathrm{P}$ o $\mathrm{K}$, la planta necesita en canticlades elevadas, las posibilidades de ser supliclos por hoja quedan limitadas por consideraciones prárticas obvias. Sin embargo se ha demostrado que las plantas algodoneras, la remolacha azucarera, el girasol y otras especies aún en condiciones normales de crecimiento y desarrollo, cuando la mayor parte de los elementos nutritivos entra en la planta a través de la raíz, el paso a través de las hojas ofrece otra fuente de nutrición (22).

Se ha demostrado que en remolacha azucarera la aplicación de $\mathrm{P}$ adicional, por hoja, en el período de acumulación de azúcar aumenta el contenido de azúcar en las raíces.

Los problemas propios de fijación del $\mathrm{P}$ en muchos suelos pueden permitir mediante los tratamientos foliares soluciones ventajosas en cleterminadas circunstancias.

Las aspersiones de $\mathrm{N}$ han quedado relegadas a las de urea con la 
que se efectúan tratamientos comerciales principalmente en manzano, como también ha sido utilizada con éxito en bananeros, caña de azúcar, viñedos, citrus y otros cultivos. Hay antecedentes de que aspersiones aéreas de urea en caña de azúcar incrementaron el tenor de $\mathrm{N}$ y clorofila de la hoja mucho más rápidamente que las efectuadas al suelo. Es evidente que el $\mathrm{N}$ así aplicado es fácilmente absorbido y movilizado por lo que en ciertos casos, su aspersión puede constituir una práctica de interés (13). Desde luego, que las consideraciones prácticas y de economía en relación a las necesidades nitrogenadas de la planta hacen insustituibles las prácticas comunes de abonamiento nitrogenado por el suelo.

Como resumen puede deducirse que la fertilización foliar está sujeta a complejas condiciones que hacen necesario el análisis especial sobre la mayor o menor conveniencia en su realización.

Los tratamientos con elementos menores son decididamente promisorios. En casos muy especiales la aspersión nitrogenada. Menos prometedores los basados en aplicaciones de $\mathrm{P} y$, en menor grado aún, los correspondientes a $\mathrm{K}$.

Por fin, y a la luz de trabajos recientes, ha quedado demostrada la estrecha relación existente entre la nutrición mineral por las partes aéreas de la planta y los procesos de fotosíntesis, respiración, actividad de los fermentos y alimentación mineral por raíz. Esta influencia sobre los grandes procesos vitales del vegetal explicaría la interrelación entre alimentación mineral de la planta a través de hoja y raíz y, en consecuencia, las ya observadas influencias de las condiciones del suelo en los resultados de los tratamientos foliares.

En cuanto a efectividad general de los tratimientos foliares KaIND ( ${ }^{1}$ ) menciona los siguientes principios:

1) Hay dos períodos en los cuales la fertilización fuliar es más efectiva: la etapa temprana y aquella cuando la vitalidar de la planta es máxima.

2) Aspersiones efectuadas por la tarde son preferibles a las realizadas por la mañana, debido a que el secado es más lento y la formación de rocío, por las mañanas, removiliza los nutrientes que permanecen en la superficie de la hoja.

3) Condiciones de alta humedad promueven la absorción foliar. Es conveniente realizar nuevas aspersiones con pequeñas cantidades de agua después de efectuados los tratamientos.

4) Una combinación de aplicación foliar y por suelo se traduce en los más altos rendimientos.

5) En los frutales tener presente la conveniencia de que sea tratada la parte inferior de las hojas.

Un agregado a lo expuesto podría ser el tener presente la posible interacción de nutrientes (o nutrientes con vehículus u otros productos que se usen en la aspersión) que como hemos visto, pueden acrecentar o disminuir la absorción según los casos. 


\section{EL ZN COMO DEFICIENCYA Y PRINCIPIOS GENERALES DE CONTROL}

El rlenominado "mottle leaf" es el síntoma característico de la deficiencia de $Z n$ en numerosas especies. En citrus especialmente $\left({ }^{20}{ }^{23}\right)$ existe amplia bibliografía como excelentes trabajos con reproducciones en fotocolor de las hojas afectadas.

En forma semejante al Fe, los tratamientos en el suelo de sale: de $\mathrm{Zn}$ no dieron los resultados esperados y en general se estima que la aspersión nutritiva es la forma más eficaz de control.

Las conclusiones obtenidas al respecto son (-4):

1) Una aspersión de Zn metálico (proveniente de sustancias como sulfato, óxido o carbonato) en dosis de 50 gramus en 100 litros de agua, condujo a un apreciable porcentaje de eficiencia.

2) Dañns ocasionados al follaje pueden ser evitados por un agente precipitante como la cal.

3) A equivalentes canticlades de Zn metálico los espolvoreos son menos eficientes.

4) A bajas concentraciones de $\mathrm{Zn}$, el uso de humectantes y adhesivos en general, aunque no siempre, aumentó los efectos del elemento. En concentraciones mayores de 20 gramos de $\mathrm{Zn}$ por 100 litros de agua, nu parece beneficiosa la acción de esos agregados.

\section{TAA DEFICIENCIA DE ZN EN CAFÉ}

Desde época relativamente reciente la acción del $Z n$ en plantas de café ha traído la atención de técnicos e investigadores.

Trabajos pablicados indican la existencia del problema en almácigos y plantaciones establecidos, dándose las bases generales para su control $\left({ }^{30}\right)\left({ }^{38}\right)\left({ }^{39}\right)\left({ }^{40}\right)\left({ }^{41}\right)$.

El mismo está basado en la aplicación de $\mathrm{Zn}$ al suelo y por aspersiones de $\mathrm{SO}_{\mathrm{Zn}} \mathrm{Z}$. Se destacan los mejores resultados de lus tratamientos foliares. La deficiencia de Zn es extendida en Costa Rica presentándose en forma esporádica y sin afectar grandes superficies $\left({ }^{43}\right)\left({ }^{40}\right)$.

Trabajos realizados sobre preferencia de absorción por raíces y hojas y su relación con la de otros elementos (Fe, $\mathrm{Mn}, \mathrm{Cu}, \mathrm{Mo}$ ) mostraron, usanrlo $\mathrm{Zn}^{65}$, que plantas de almácigo, llevadas a soluciunes nutritivas absorbieron una mayor proporción le $\mathrm{Zn}^{65}$ por las hojas y también la influencia de los factores $\mathrm{Mn}, \mathrm{Cu}$ y $\mathrm{Mo}$ en la cantidad absorbida ( $\left.{ }^{17}\right)$.

La aplicación de quelatos metálicos al suelo o soluciones nutritivas ( $\left.{ }^{21}\right)$ mostró interesantes efectos en café aún en plantas sin síntomás visibles de deficiencia. 
El estado actual cle las investigaciones, a su vez indica, que la aplicación de quelatos al suelo está reservada para cultivos de gran valor (6). En la mayoría de los casos siguen siendo recomendables las aspersiones nutritivas. Por lo tanto, interesa conducir los estudios hacia los factores que pueden influenciar la absorción y traslación de un elemento.

\section{OBJETIVOM DEL TRABAJo REALIZADO}

Dentro de su naturaleza preliminar es objetivo clel trabajo obtener una orientación sobre los factores que pueden influenciar la a.bsorción y traslación del Zn en plantas jóvenes de café.

\section{Matertales y Métonos}

Se trabajó con plantas de café (Coffea arabiga var. Borbon salvadoreño) provenientes de una siembra efectuada en Septiembre de 1958 y transplantadas a macetas en marzo de 1959 , siendo tratadas aproximadamente un mes después.

La tierra utilizada provino de una zona vecina al Instituto en cuyas proximidades hay especies con sintomas de deficiencia de $\mathrm{Zn}$. La misma fué homogeneizada y se introdujo en macetas de barro.

Los tratamientos fueron basarlos en la aplicación de una dosis única de $Z n$, a una hoja, observanclo variaciones en la absorción y traslación según diversos factores.

La dosis aplicada consistió en $117 \mathrm{mg}$ de $\mathrm{Zn}$ por hoja tratarla. A tal eferto se calibró un gotero que mediante 3 gotas permitirá lis indicarda dosis.

Los tratamientos fueron:

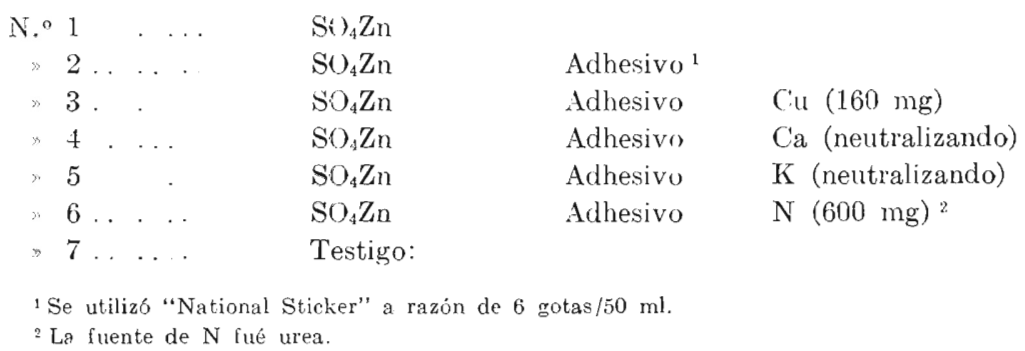

Las soluciones fueron marcadas con $\mathrm{Zn}{ }^{65}$. isótopo radioactivo del Zn existente en la naturaleza.

Las características del $\mathrm{Zn}^{65}$ utilizado son: Vida media 265 días, obtenido en la pila atómica de Oak Ridge (USA) con las características Zn-65-P, procesado con alta actividad especifica, 75 me por $\mathrm{g}$ de $\mathrm{Zn}$ y una concentración de $1 \mathrm{mc} / \mathrm{ml}$ al estado de $\mathrm{Cl} . \mathrm{Zn}$ en solurión en $\mathrm{HCl}$. $\mathrm{Al}$ proceso de producrión sigue el esquema $\mathrm{Zn}^{64}(\mathrm{n}, \gamma)$ 
$\mathrm{Zn}^{65}$ es decir, que el $\mathrm{Zn}^{64}$ bombardeado con neutrones se transforma en $\mathrm{Zn}^{65}$ emitiendo radiación gamma con una energía de $1,12 \mathrm{M}$. E. V. y desintegración beta con una energía de 0.325 M. E. V.

Las soluciones utilizadas fueron marcadas con 100 microcurios: por mililitro y de acuerdo a la closis aplicada $\left(3\right.$ gotas $=\frac{1}{10}$ de ml) resultaron 10 microcurios por hoja tratada.

Cada tratamiento consistió en 5 plantas de las que se eligió una hoja ubicarla en su parte superior y en similar posición.

Se realizó un primer tratamiento para observar el efecto de altas concentraciones por unidad de área foliar aplicándose 3 gotas por hoja $\left(^{5}\right)$.

Se cosecharon a los 10 días torlis las hojas tratadas y se procedió a desenterrar dos plantas de cada tratamiento. Hojas y plantas se lavaron con solución rletergente acidificada y posteriormente con Versene al $3 \%$.

Lats observaciones se resumen en el siguiente cuadro:

\begin{tabular}{|c|c|c|c|c|}
\hline $\begin{array}{l}\text { Tra- } \\
\text { tamiento }\end{array}$ & $\begin{array}{l}\text { c. p. m. } \\
\text { hojas } \\
\text { cosechadas ' }\end{array}$ & c. p. m. plantas 1 & $\begin{array}{l}\mathrm{mr} / \text { hora } \\
\text { hojas } \\
\text { tratadas }\end{array}$ & Observaciones \\
\hline 1 & 940 & $\begin{array}{l}\text { Hojas } 110 \\
\text { Tallo } 150 \\
\text { Raíz } 120\end{array}$ & 0,5 & Necrosis por quemaduras. \\
\hline 2 & $\begin{array}{r}900 \\
-\quad 9\end{array}$ & $\begin{array}{l}\text { Hojas } 110 \\
\text { Tallo } 130 \\
\text { Raíz } 120\end{array}$ & 0,5 & Necrosis por quemaduras. \\
\hline 3 & 730 & $\begin{array}{l}\text { No se apreciaron } \\
\text { diferencias con el } \\
\text { fondo. }\end{array}$ & 0,5 & $\begin{array}{l}\text { Muy leve necrosis. Hoja } \\
\text { ondulada. }\end{array}$ \\
\hline 4 & 520 & $\begin{array}{l}\text { Hojas } 110 \\
\text { Tallo } 130 \\
\text { Raíz } \\
130\end{array}$ & 0,5 & $\begin{array}{l}\text { Se destacó la hoja trataria } \\
\text { por un color verde intenso. }\end{array}$ \\
\hline 5 & 360 & $\begin{array}{l}\text { No se apreciaron } \\
\text { difereneias con el } \\
\text { fondo. }\end{array}$ & 0,5 & $\begin{array}{l}\text { Se notó cierta gcmosidat } \\
\text { en las hojas tratadas. }\end{array}$ \\
\hline 6 & 400 & $\begin{array}{l}\text { No se apreciaron } \\
\text { diferencias con el } \\
\text { fondo. }\end{array}$ & 0,5 & \\
\hline
\end{tabular}

1 Integrador mod. 1620 A Nuclear Chicago (Geiger-Muller).

${ }^{2}$ Detector mod. 1612 Nuclear Chicago (Geiger-Muller). 
Ante la evidencia de que las altas concentraciones usadas en áreas pequeñas habían producido quemaduras se efectuó, una vez eliminadas las hojas tratadas, una segunda aplicación en otra hoja.

La misma consistió en igual dosis de $\mathrm{Zn}$ y con prácticamente igual radioactividad pero en la parte inferior dispersando esta vez con un pincel la solución.

A los diez días se cosecharon las hojas, desenterraron las plantas procediéndose al lavado en la forma ya especificada.

El cuadro de observaciones es el siguiente:

\begin{tabular}{|c|c|c|c|c|}
\hline $\begin{array}{c}\text { Triz- } \\
\text { tamiento }\end{array}$ & $\begin{array}{c}\text { c, p. m. } \\
\text { hojas } \\
\text { cosechadas } 1\end{array}$ & c, p. m. plantas 1 & $\begin{array}{l}\mathrm{mr} / \text { hora } \\
\text { hojas } \\
\text { tratadas } 2\end{array}$ & Observaciones \\
\hline 1 & 950 & $\begin{array}{l}\text { Hojas } 150 \\
\text { Tallo } 150 \\
\text { Raíz 160) }\end{array}$ & 0,5 & Buen estado general. \\
\hline 2 & 800 & $\begin{array}{l}\text { Hojas } 140 \\
\text { Tallo } 140 \\
\text { Raíz } 140\end{array}$ & 0,5 & Buen estado general. \\
\hline 3 & 700 & $\begin{array}{l}\text { Hojas } 130 \\
\text { Tallo } 130 \\
\text { Raíz } 140\end{array}$ & 0,5 & Buen estado general. \\
\hline 4 & 150 & $\begin{array}{l}\text { Hojas } 110 \\
\text { Tallo } 110 \\
\text { Raíz } 130\end{array}$ & 0,5 & Buen estado general. \\
\hline 5 & 150 & $\begin{array}{l}\text { No se apreciaron } \\
\text { diferencias con el } \\
\text { fondo. }\end{array}$ & 0,5 & Buen estado general. \\
\hline 6 & 150 & $\begin{array}{l}\text { No se apreciaron } \\
\text { diferencias con el } \\
\text { fondo. }\end{array}$ & 0,5 & Buen estado general. \\
\hline
\end{tabular}

1 Integrador modelo 1620 A Nuclear Chicago (Geiger-Muller)

${ }^{2}$ Detector modelo 1612 Nuclear Chicago (Geiger-Muller).

Las radiautografías tomadas fueron realizadas con la técnica de aposición sencilla ("6), y se utilizó película Kodak, Blue Brand. El tiempo de exposición se calculó en 3 días para las hojas tratadas y 9 días para el resto de la planta.

Las radiautografías de los tratamientos en la cara superior de 
las hojas revelaron las quemaluras, por otra parte ya visibles y una tralación muy débil hacia la parte inferior del tallo.

Las correspondientes al tratamiento de la hoja, parte inferior, revelaron mayor intensidad en los tratamientos 1 y 2 y en general pora uniformidad en la absorción.

Respecto a la traslación se observó con mayor intensidacl en el scetor inferior del tallo.

A los efectos de efectuar contaje y eventualmente análisis químico se procedió a la digestión húmeda del material ( $\left.{ }^{34}\right)$ utilizando ácidos nítrico, perclórico y sulfúrico, concentrados en las proporciones 10-4-1 y a razón de $5 \mathrm{ml}$ por gramo de materia seca.

Se llevó a volumen y procedió al contaje en contador Nuclear Chicago, modlelo $161 \mathrm{~A}$ (Geiger-Muller), llevándose los cálculos a c. p. m. por $100 \mathrm{mg}$ de materia seca, en el caso de las hojas tratadas, y por $500 \mathrm{mg}$ de materia seca en lo que al resto de la planta se refiere. El sistema utilizado fué sumergir el tubo Geiger-Muller dentro del líquido ubicado en un tubo de centrífuga. Se constató que $100 \mathrm{mg}$ de materia seca llevados a $10 \mathrm{ml}$ daban el mismo número de cuentas que el mismo peso llevado a $20 \mathrm{ml}$ por lo que la pérdida en actividad específica era proporcional a la ganancia por geometría al permitirse, con el mayor volúmen, un mejor contacto con el tubo.

A continuación se resumen los resultados:

\begin{tabular}{|c|c|c|}
\hline Tratamiento & $\begin{array}{l}\text { c. p. m. hojas } \\
\text { tratadas }^{1}\end{array}$ & $\begin{array}{l}\text { c. p. m. resto de } \\
\text { la planta }{ }^{2}\end{array}$ \\
\hline 1 & 434 & 175 \\
\hline 2 & 780 & $1+9$ \\
\hline 3 & 379 & 170 \\
\hline$t$ & $27 t$ & 160 \\
\hline$\check{5}$ & 366 & 166 \\
\hline bi & $\begin{array}{c}\text { cara inlerior } \\
256 \\
\text { cara superior } \\
190\end{array}$ & $\begin{array}{l}172 \\
114\end{array}$ \\
\hline
\end{tabular}

1 Calculaclus sobre la base de $100 \mathrm{mg}$, secus llevados luego cle digestión a 10 mal.

- Calculados sobre la base de $500 \mathrm{mg}$. secos llevados luego de digestión a $50 \mathrm{ml}$. 


\section{1)ISCUSIÓN}

Las aplicaciones a concentraciones elevadas conviene sean efectuadas en forma que asegure un esparcido uniforme, principalmente cuando por razones de ácidos libres o reacción en general sean posible quemaduras o áreas necróticas que dificultan la interpretación de resultados.

En base al tratamiento $N .{ }^{\circ} 6$, que en su aplicación superior no produjo necrosis, comparando con la inferior, se observa mayor absorción y traslación como consecuencia del tratamiento efectuado en la parte inferior de la hoja.

Se confirmaría el concepto de mayor alssorción por el envés de la hoja.

En todos los contajes se observa que la absorción fue influenciada por la presencia de otros elementos. Los 1 y $2\left(\mathrm{SO}_{4} \mathrm{Zn}\right.$ y $\mathrm{SO}_{4} \mathrm{Zn}$ con adhesivo) denotan mayor absorción.

Todos los agregados disminuyeron la absorción pero, en cuanto a la tralsación pareciera no existir influencia de los mismos pues en todos los tratamientos y comparando entre los mismos no hubo diferencias significativas.

El movimiento del $\mathrm{Zn}$ dentro de la planta se habría producido en una escala muy reducida.

Las características del $\mathrm{Zn}^{65}$ con su débil radiación gamma harían aconsejable la utilización de contador de centelleo para un eficiente contaje.

En cuanto a la digestión convendría proceder a la inisma sin $\mathrm{H}_{2} \mathrm{SO}_{4}$ pues existe el problema de la evaporación en caso que se deseen analizar muestras sólidas.

\section{CONCLUSIONES}

1. Conveniencia de un esparcido uniforme de la solución nutritiva en el tratamiento foliar.

2." Importancia del adhesivo a utilizar.

3." Todos los agregados usados como neutralizantes, cobre e incluso nitrógeno, disminuirían la absorción.

4. El Zn se moviliza en cantidades muy reducidas y esta traslación no se vió afectada por la cantidad absorbida.

5. ${ }^{\circ}$ La traslación de elementos como el Zn no sería función de la cantidad absorbida.

6. ${ }^{\circ}$ Los factores estudiados influyeron en la absorción pero no en la traslación. 


\section{RESUMEN}

Se efectúa una revisión sobre los principios cle influencia en los tratamientos fertilizantes por las partes aéreas de las plantas.

Por medio de aplicaciones de $\mathrm{Zn}{ }^{65}$ en plantas jôvenes de café y siguiendo técnicas de radiautografía $y$ contaje de elementos radiactiros se efectúan obserraciones sobre la absorcion del Zn y factores que puedan influenciar la misma y su posterior transporte a otros organos de la planta.

Dentro del carácter preliminar de la investigación se destacan algunas conclusiones como ser: importancia del esparcido uniforme, influencia del adliesivo, efecto de otros agregados en la absorción y presumible influencia en el traslarlo.

Se destaca la importancia de los tratamientos arreos con fertilizantes $y$ la necesidad de estudios destinados a investigar su relación e influencia sobre la absorción de elementos por raíz.

\section{SUMMAR}

The objective of this work is to siuly the absorption and trasluation of $\mathrm{Zn}$ from SOZ $\mathrm{Zn}$ under diverse conditions. It has been started with the application of one dosis of SOAn tagged with Zn ${ }^{n, 5}$, to coffee seedlings under crontrolled conditions of acidaty and interaction with other elements (Ca, N, K and $\mathrm{Cu}$ ).

The work is of preliminary nature with the objective of seeking clarification of those factors that have more influence on the absortion and iraslocation of $\mathrm{Zn}$ in coffee plants.

\section{Bibtiografít}

(1) Kand, K. - Foliar fertilization with fofote nutrient labelled with $p^{3 !}$. Proc of 2nd. Radioisotope Conference, Oxford, 1954, 347-40t.

(2) Tukey, H. B., Wrtwer, S. H., Teubner, F. \&. \& Lone, W. G. - Utilización de los isótopos radiactivos en el estudio de grado de efectividad de la absorción foliar de los elementos nutritivos para las plantas. Actas de la Conferencia de Gincbra. 12, 15t-16i, 1956.

(3) Tuker, H. B., Thanon, R. L., Hinsvark, O. N., \& Wittweir, S. H. Absorption of nutrients by stems and branches of wooly plants, Science, 116: 167-8, 1952.

(t) Witrwer, S. H. - Nutrient uptake with special reference to foliar absorption. Atomic energy and Agriculture. Atlanta, 1057, ed. C. L. Comar.

(5) Wallihin, E. F. and Hexmann-Herschegro, L. - Some factors affecting absorption and translocation of $Z_{n}$ in citrus plants. Plant Physiology 31: (t) 321-t.

(6) Walcace, A. and Benrt, A. A. - Irun and Zine Foliage Sprays: radioge- 
tive trarers used in basic studies on factors influencing absorption and translocation: of mieronutrients. California Agriculture, March, 1958.

(7) Terbner, F. ( ., Witwwer, S. H., Tuckey, H. B. \& Long, W. C. - Factors affecting the uptake of foliar applied radionucleides. Michigan Agric. Exp. Sta. Bull. 39: 398-415, 1957.

(8) Gustafson, F. G. - Absorption of $\mathrm{Co}^{60}$ by leaves of young plants and it translocation throngh the plant. Amer. Jou. Bot 43-157-160. 1956.

(9) Volk, R. \& Arimfre, C. Mc. - Factors affecting the foliar aboorption of N15 labelled urea by tobacco. Proc. Soil. Sc. Amêr. 18: 308-12. 1954.

(10) Gustafson, F. G. - Comparative absorption of ( $0^{60}$ by upper and lower epidermis of leaves. Plant Physiology 32: 141-2, 1957.

(11) Crafss, A. S. - Sulfuric acid as a penetrating agent in arseniral spray: for weerl control. Hilgardia 8: 125-17, 1953.

(18) Robertis, F. ․, Sorthwick, M. D. and Palmiter, D. H. - A mimrochemical examination of Mac Intosch apple leaves. Plant Physiol. 23: $557-9$.

(13) Bornon, D. - Nutrition by foliar application. Rev. Plant Fhys. 5: 31-51, 1954 .

(14) Van Overbeer, J. - ibsorption and transloration of plants regulators. Ann. Rev. Plant Physiol. 7: 355-72. 1956

(15) Diccionario de Botinica. Ed. Labor.

(16) EsAU, K. - Plant Anatomy. 1953.

(17) Malavotta, F., Anzolda, J. P., HaAc, H. P. - Preliminary note on the absorption of radiozine by young cofee plants (Coffea arabiga L.) grown in mutrient solution. Phyton s: 1-6, 1950.

(19) Dedecca, D. M. - Recientes progresos en muestro vonocimiento del inbol de café. IV Anatomáa. Reimpresión I.I.C.A. 1958.

(19) Bidduzper, 0. - Translocaticn of radioactive mineral nutrients in plants. A Conference on the Use of Isolopes in Ilant and Animal Research. Kansa: St. Coll., p. 48, 1953.

(20) Wallace, T. - The diagnosis of mineral defieiences in plants. Ed. by Chemical Publishing Co. Inc. New York. 1953.

(1) Stewart, L, and Leonard, ( . 1). - Chelater metal for growing plant: Mineral Nutrition of iruit Crons. Ed por N. F. Chillers. 1954 (775-803).

(22) KLechкоwsкi, V. M. - T'so de trazadores en estudios sobre la apliarión de fertilizantes. Actas de la Conferencia de Ginebra. 12: 122-31, 1956.

(23) Camp, A. F., Chapman, H. D. and Parker, E. R. - Symptoms of citü malnutrition. Hunger Signs in Crops. 1949.

(24) Parker, E. R. Proc. Am. Soc. Hort. 35: 217-26. 1938.

(25) Bibliography of the Literature on the Minor Elements and Their Relation to Plant and Animal Nutrition, 1955. Vol. IV.

(26) Comar, C. L. Radioisotopes in Biology and Agriculture, 1955.

(27) Conlma Hale y Mrbuer, E. J. - Determination of small Amumts of Zine in Plant Materials. Industrial and Engineering Chemistry, March 1941.

(28) Culot, J. P. et Van Vambeke, A. - Contribution a l'Etude des Défi. ciences Minérales du cafeier d'Arabie au Kivu. 1958. 
(29) Dean, L. A. - Aplicaciones de los isótopos radioactivos al estudio de suelos y fertilizantes, Naciones Unidas, Vol. XII, 1956.

(30) Franco, C. M. y Mendes, H. C. - Deficiencias de microelementos en caffeiro (Nota preliminar). Bol. Super. Scrn. Café 28.

(31) González, C. y Camacho, J. Síntomas de la deficiencia del boro en el cafeto. Informe Pretiminar. Bolctín Técnico N. ${ }^{\circ} 11, M$. A. I., Costa Rica. 1952.

(32) Hemen, Eurich J. and Benne, Erwin J. - Report on Zine in Plants. - Joumal of the Associution of Official Agricullueal Chemists. Vol. 3t, N. 3, prig. 692. 1951.

(33) Hentwals, J. B. - Reaction of Ferric Ethylencramine Tetraacetate with Soil Clay Minerals. S. Sc. Sept. 1058.

(3-1) JACKeon, M. L. - Soil Chemical Analysis, 1958.

(35) LotT, W. L., Nepy, J. P., Gallo, J. R., Medcalf, J. C. - Ia Ténica del anílisis foliar en los estudios del cafe. IBEC N.' 11, 1956.

(36) Młchıdo, A. - Coffee Fertilizers and Foliar Diagnosis. Bol. Inf. Chimchinú $N .^{\circ} 7,1956$.

(37) Medcalf, J. C. e Lox', W. L. - Aplicagro de quelatos metálicos en caffeiros, IBEC. Research Institute, N. ${ }^{11}, 1956$.

(38) Montero, J. M. - La deficiencia del uinc: en los almárigos de café. Suclo Tico N.०28. 1952.

(39) Mowry, H. - Minor Element Defficiences in Coffee in Costa Rira. Forcign Agriculture, May 1953.

(40) Murcer, L. E. - Mineral Defficiences in Coffee in Costa Rica, 1954.

(41) Peralta, Marí Ti. - Análisis de alumos minerales eq hojas de café con y sin afección de cafe "macho". Tesis IICA, Agosto 1952.

(42) Piper, ('. S. - Soil and Plant Analysis. 1944.

(43) Pérez, Vícror, M. - Algmas cleficiencias minerales del cafeto en Custa Rica. STICA, Bol. Trf. N. 2, 1957.

(44) Villafuerte Paredis, J. V. - Algunos distubios nutricionales del cafeto en Costa Rica. Tesis I/CA, 1054. 